HEAT TRANSFER - ASIAN RESEARCH

EDITOR-IN-CHIEF: PROFESSOR WILLIAM. M. WOREK -TEXAS A\&M UNIVERSITY-KINGSVILLE, TEXAS, USA.

PUBLISHER- WILEY USA

ONLINE ISSN: 1523-1496

Accepted August $13^{\text {th }} 2019$

\title{
INFLUENCE OF VARIABLE VISCOSITY AND THERMAL CONDUCTIVITY, HYDRODYNAMIC AND THERMAL SLIPS ON MAGNETOHYDRODYNAMIC MICROPOLAR FLOW: A NUMERICAL STUDY
}

\author{
M.A. Rahman ${ }^{1}$, M.J. Uddin ${ }^{2}$, O. Anwar Bég ${ }^{3}$ and Ali Kadir ${ }^{3}$ \\ ${ }^{1}$ Natural Science Group, National University, Gazipur-1704, Bangladesh. \\ ${ }^{2}$ Mathematics Department, Faculty of Science and Technology, American International \\ University-Bangladesh, Kuratoli, Dhaka 1229, Bangladesh. \\ ${ }^{3}$ Aeronautical and Mechanical Engineering Department, School of Computing, Science \\ and Engineering, Newton Building, University of Salford, M54WT, UK.
}

\begin{abstract}
:
Thermophysical and wall slip effects arise in many areas of nuclear technology. Motivated by such applications, in this article the collective influence of variable viscosity, thermal conductivity, velocity and thermal slips effects on a steady twodimensional magnetohydrodynamic microplar fluid over a stretching sheet are analyzed numerically. The governing nonlinear partial differential equations have been converted into a system of non-linear ordinary differential equations using suitable coordinate transformations. The numerical solutions of the problem are expressed in the form of non-dimensional velocity and temperature profiles and discussed from their graphical representations. Nachtsheim-Swigert shooting iteration technique together with the sixth order Runge-Kutta integration scheme has been applied for the numerical solution. A comparison with the existing results has been done and an excellent agreement is found. Further validation with adomian decomposition method is included for the general model. Interesting features in the heat and momentum characteristics are explored. It is found that greater thermal slip and thermal conductivity elevate thermal boundary layer thickness. Increasing Prandtl number enhances Nusselt number at the wall but reduces wall couple stress (micro-rotation gradient). Temperatures are enhanced with both magnetic field and viscosity parameter. Increasing momentum (hydrodynamic) slip is found to accelerate the flow and elevate temperatures.
\end{abstract}

Key words: Magnetic field, Variable thermal conductivity, Velocity slip; Thermal slip, Nuclear thermal transport; Micropolar fluids; Boundary layers; Numerical solutions.

*Corresponding author: Email: jashim_74@yahoo.com 


\section{NOMENCLATURE}

a

B

$B_{0}$

$C_{1}$

$C f_{x}$

$c_{p}$

$d$

$E_{1}$

$f$

$g$

$h(x)$

$j$

$k$

$k_{\infty}$

$l$

M

$M_{w}$

$n$

$N_{1}$

$N u_{x}$

$\operatorname{Pr}_{\infty}$

$\operatorname{Re}_{x}$

$T$

$T_{w}$

$T_{\infty}$

$u$

$u_{w}(x)$

$x$

$y$

Stretching rate based on surface velocity, $\left[\mathrm{s}^{-1}\right]$

Magnetic induction, [Wb. $\mathrm{m}^{-2}$ ]

Uniform magnetic field strength, [Wb. $\mathrm{m}^{-2}$ ]

Constant [-]

Local skin-friction coefficient [-]

specific heat of the fluid at constant pressure, $\left[\mathrm{J} \cdot \mathrm{kg}^{-1} \cdot \mathrm{K}^{-1}\right]$

Thermal slip parameter [-]

Thermal slip factor, [m]

Dimensionless stream function [-]

Dimensionless micro-rotation [-]

Local heat transfer coefficient $\left[\mathrm{W} /\left(\mathrm{m}^{2} \bullet \mathrm{K}\right)\right]$

Micro-inertia density, $\left[\mathrm{m}^{2}\right]$

Temperature dependent thermal conductivity of the fluid, $\left[\mathrm{W} \cdot \mathrm{m}^{-1} \cdot \mathrm{K}^{-1}\right]$

Constant undisturbed thermal conductivity $\left[\mathrm{W} \cdot \mathrm{m}^{-1} \cdot \mathrm{K}^{-1}\right]$

Velocity slip parameter [-]

Magnetic field parameter [-]

Wall couple stress $\left[\mathrm{N} / \mathrm{m}^{2}\right]$

Micro-rotation parameter [-]

Velocity slip factor, $\left[\mathrm{s} . \mathrm{m}^{-1}\right]$

Local Nusselt number [-]

Constant Prandtl number [-]

Local Reynolds number [-]

Temperature, $[\mathrm{K}]$

Temperature at the surface, $[\mathrm{K}]$

Temperature of the ambient fluid, $[\mathrm{K}]$

Velocity along $x$-axis, $\left[\mathrm{m} . \mathrm{s}^{-1}\right]$

Velocity of the sheet, $\left[\mathrm{m} . \mathrm{s}^{-1}\right]$

Axis in direction along the sheet, [m]

Axis in direction normal to the sheet, [m]

\section{Greek Symbols}

$\begin{array}{ll}v_{s} & \text { Spin-gradient viscosity, }\left[\mathrm{m}^{2} \cdot \mathrm{s}^{-1}\right] \\ v_{\infty} & \text { Kinematic viscosity at constant property, }\left[\mathrm{m}^{2} \cdot \mathrm{s}^{-1}\right] \\ \mu & \text { Coefficient of dynamic viscosity, [Pa.s] } \\ \tau_{w} & \text { Surface shear stress, [Pa] } \\ \mu_{\infty} & \text { Coefficient of dynamic viscosity with constant property [Pa.s] } \\ \rho_{\infty} & \text { Mass density of the fluid, }\left[\mathrm{kg} \cdot \mathrm{m}^{-3}\right] \\ \sigma & \text { Micro-rotation component normal to the } x y \text {-plane } \\ \sigma_{0}^{\prime} & \text { Electrical conductivity, }\left[\mathrm{m} \Omega \mathrm{m}^{-1}\right] \\ \sigma_{0} & \text { Magnetic permeability, }\left[\mathrm{N} \cdot \mathrm{A}^{-2}\right]\end{array}$




$\begin{array}{ll}\theta & \text { Dimensionless temperature }[-] \\ \theta_{r} & \text { Variable viscosity parameter [-] } \\ \Delta & \text { Vortex viscosity parameter }[-] \\ \xi & \text { Spin-gradient viscosity parameter } \\ \psi & \text { Stream function, }\left[\mathrm{m}^{-2} . \mathrm{s}^{-1}\right] \\ \eta & \text { Similarity variable }[-] \\ \Delta \eta & \text { Step size } \\ \text { Super Scripts } & \\ ()^{\prime} & \text { Ordinary differentiation with respect to } \eta \\ \text { Sub Scripts } & \\ w & \text { Conditions at the surface } \\ \infty^{+} & \text {Free stream conditions }\end{array}$

\section{INTRODUCTION}

Heat transfer is a fundamental aspect of many nuclear engineering transport processes. It may arise in any three of the familiar modes (conduction, convection and radiation) and indeed these modes often arise simultaneously. Interesting applications include phase change saturated nucleation ( see Uesawa et al., 2017), transient electrically-conducting convection flows of liquid sodium (Mukhopadhyay, 2011), nuclear propulsion systems cooling (Akyuzlu, 2015), conjugate thermal transport (Corradini, 2003), super-critical thermal convection in rod bundles (Gradecka et al., 2016), heat emission in turbulent flows of mercury, sodium, lead-bismuth and sodium-potassium liquid metals (Kirillov, 2016) and hybrid electrolysis systems exploiting nuclear energy (Harvego et al., 2010).

Electromagnetic flows also arise in nuclear power systems wherein magnetic fields are deployed to control high temperature electrically conducting plasmas from damaging for example channel walls. Magnetohydrodynamic concerns the interaction between electrically conducting liquids and applied magnetic fields. It has extensive applications in emergency heat removal in fast reactors (Bogdanova et al., 2017), molten metal pumps for rapid removal of heat from cores (Doležel et al., 2009) and feeder wall thinning mechanisms in flow-assisted corrosion of alloys in nuclear reactor channels (Kim et al., 2005). Although often working fluids in nuclear reactor systems are air and water, many non-Newtonian liquids are increasingly being deployed. Such fluids cannot be simulated with the classical Navier-Stokes viscous fluid model based on the Newtonian viscosity law. Important characteristics exhibited by non-Newtonian fluids (Schowalter, 1978) include variable shear stress-strain characteristics, yield stress, stress relaxation, 
elongation, memory and micro-structural behaviour associated with particle-laden suspensions. Many elegant formulations have been deployed to simulate non-Newtonian flows in chemical, nuclear and mechanical engineering, with and without heat transfer including Reiner-Rivlin differential fluids (Hayat et al., 2012), Maxwell upper convected viscoelastic fluids (Bhatti et al., 2017), Walters-B fluids (Bég et al., 2010), Casson viscoplastic fluids (Akbar et al., 2016) etc. These models however do not offer a robust framework for simulating micro-structural features of suspensions e.g. particle-doped coolants in nuclear reactors. Eringen (1966) introduced the theory of micropolar fluids as an extension of the Navier-Stokes model to describe suspensions containing particles. Micropolar fluids are a simplification of Eringen's more general micro-morphic fluid theory (Eringen, 1964) in which isotropic, polar properties are assumed and deformation of suspended molecules (micro-elements) is neglected. Physically, they represent fluids with molecules which are able to rotate independently of the fluid stream flow and local vortices. These fluids provide a good approximation for capturing many sophisticated features exhibited by real fluids in nuclear, mechanical, chemical and even medical engineering. Micropolar fluids contain micro-constituents that can undergo rotation i.e. can sustain gyratory motions, which influence the global hydrodynamics of the flow and manifests in distinctly non-Newtonian characteristics. Micropolar flow models are formulated in terms of the linear velocity vector and the angular velocity vector (micro-rotation) associated with each particle in the fluid medium. The micro-rotation vector simulates the gyratory motion (rotation) in an average sense of the particles centered in a small volume element about the centroid of the element. Micropolar fluids have found applications in liquid crystals, lubricants, coolants, propellants, physiological suspensions (synovial liquid, blood, plasma, bile), colloidal solutions, polymeric fluids, plasmas, additives, adhesives, coating liquids etc. A good perspective of micropolar fluid dynamics with applications is given in Ariman et al. (1974) and also more recently in Eringen (2001). The elegance of micropolar fluid mechanics has allowed its deployment in boundary layer, fully developed and many other flows. Micropolar heat transfer has also emerged as a significant area of investigation. Jena et al. (1981) presented similarity solutions for natural convection boundary layer flow of micropolar fluid. Rees and Bassom (1996) investigated the Blasius flow of a micropolar fluid with finite difference methods. Gupta 
et al. (2015) used a finite element method to study stagnation micropolar heat transfer from a stretching sheet with transpiration. Ishak et al. (2006) studied the Schneider problem for micropolar fluids. Other studies of micropolar transport phenomena include Khedr et al. (2009) who considered magnetohydrodynamics, Ashraf and Batool (2013) on stretchable disk magnetic convection, Reddy et al. (2014) on reactive, dissipative hydromagnetic flow from an extending sheet. Further investigations have addressed squeeze film magnetic lubrication (Bég et al., 2012), magneto-micropolar flow from a curved stretching sheet (Naveed et al., 2016), geological plume dynamics (Bég et al., 2016), gravity-driven thin film magnetic flows (Bég et al., 2010) and entropy generation in annular micropolar flows (Jangili et al., 2016).

In many systems (including nuclear power generation) slip effects may arise at boundaries. These are generally ignored in the Navier-Stokes viscous Newtonian model. However, they cannot be neglected when non-adherence arises related to molecular properties of the gas/liquid in contact with a solid surface. Slip flows may be of the hydrodynamic or thermal type. They are present also in micro-scale electro-osmotic devices (Satapathy et al., 2010), rotating magnetofluid dynamic disk generators, optically-thick hydromagnetic flow (Bég et al., 2011), bio-nano-polymer flows (Latiff et al., 2015), additive fluoropolymer reinforced plastic fabrication (Stewart et al., 1993) and sliding lubrication (Ismail and Sarangi, 2013). Rahman et al. (2016) investigated the nanofluid flow with higher order slip from a flat plate. Mukhopadhyay (2011) derived analytical solutions for time-dependent mixed thermal convection boundary layer slip flow from a permeable extending sheet. Prasad et al. (2012) utilized a finite difference method to compute the heat and momentum transfer in enrobing boundary layer slip flow from a cylinder with a viscoplastic model. Vocale et al. (2015) computed the first order velocity and thermal slip effects on hydrodynamically and thermally fully developed gas flow through elliptical micro-channels, noting the inverse relationship between wall heat transfer rate (Nusselt number) and slip Knudsen number. Lahjomri and Oubarra (2013) used a local non-similarity method to simulate the laminar incompressible thermal convection slip flow from an isothermal surface, also observing a decrease in Nusselt number with slip effect. Uddin et al. (2016) employed a Maple boundary value solver to 
analyse anisotropic momentum, thermal, solutal and micro-organism slip effects on nanofluid bioconvection stagnation flows. Khader and Megahed (2014) used Zhou's DTM approach to compute higher order slip effects on dissipative thermal convection boundary layer flow from a porous extending sheet in porous media. Heat transfer behavior is significantly influenced also by thermo-physical properties. Dynamic viscosity and thermal conductivity in most mathematical models are assumed to be linear or inverse functions of temperature. Viscosity variation has been studied by Lai and Kulacki (1990) for thermo-solutal transport in porous media. Huda et al. (2017) investigated peristaltic pumping of nanofluids with viscosity variation in a cylindrical conduit. Modather et al. (2012) presented computational solutions for micropolar convection flow from a moving plate with viscosity variation and heat source. Thakur and Hazarika (2015) studied the collective effects of viscosity and thermal conductivity variation in hydromagnetic transient natural convective flow in porous media. Khan et al. (2016) illustrated non-aligned MHD stagnation point flow of variable viscosity nanofluids past a stretching sheet. Makinde et al. (2017) examined stagnation point flow of MHD chemically reacting nanofluid over a stretching convective surface. Kumar et al. (2018) studied magneto-convective heat transfer in mcropolar nanofluid over a stretching sheet with non-uniform heat source/sink. Sarojamma et al. (2018) presented flow of a micropolar nanofluid through a vertical channel with porous collapsible walls. Mahanthesh et al. (2018) presents numerical study of magnetohydrodynamic threedimensional flow of nanofluids with slip and radiation effects. These studies however did not consider the combined effects of momentum slip, thermal slip, variable viscosity, magnetic field and thermal conductivity on magnetohydrodynamic convection of a micropolar fluid from a vertically translating plate. This is the focus of the present article. The normalized conservation equations for mass, linear momentum, angular momentum and energy are solved with appropriate boundary conditions using higher order numerical quadrature (Uddin et al., 2016). Computations are validated with an Adomian decomposition method (ADM) (Adomian, 1994). Additional validation of special cases of the general model is conducted based on published results. The study is relevant to near-wall thermo-fluid dynamics of magnetically controlled convection in nuclear reactor flows. 


\section{MATHEMATICAL FORMULATION}

Steady two-dimensional magnetohydrodynamic (MHD) forced convection laminar boundary layer flow of a viscous incompressible and electrically conducting micropolar fluid from a horizontal plate is considered. First order hydrodynamic slip and thermal slip are present at the wall. Viscous dissipation and thermal dispersion and stratification effects are ignored. Magnetic Reynolds number is sufficiently small to negate induced magnetic field produced by the motion of the conducting fluid. Ohmic heating (Joule dissipation) and Hall current effects are also ignored. The flow model with associated coordinate system are depicted in Fig.1.

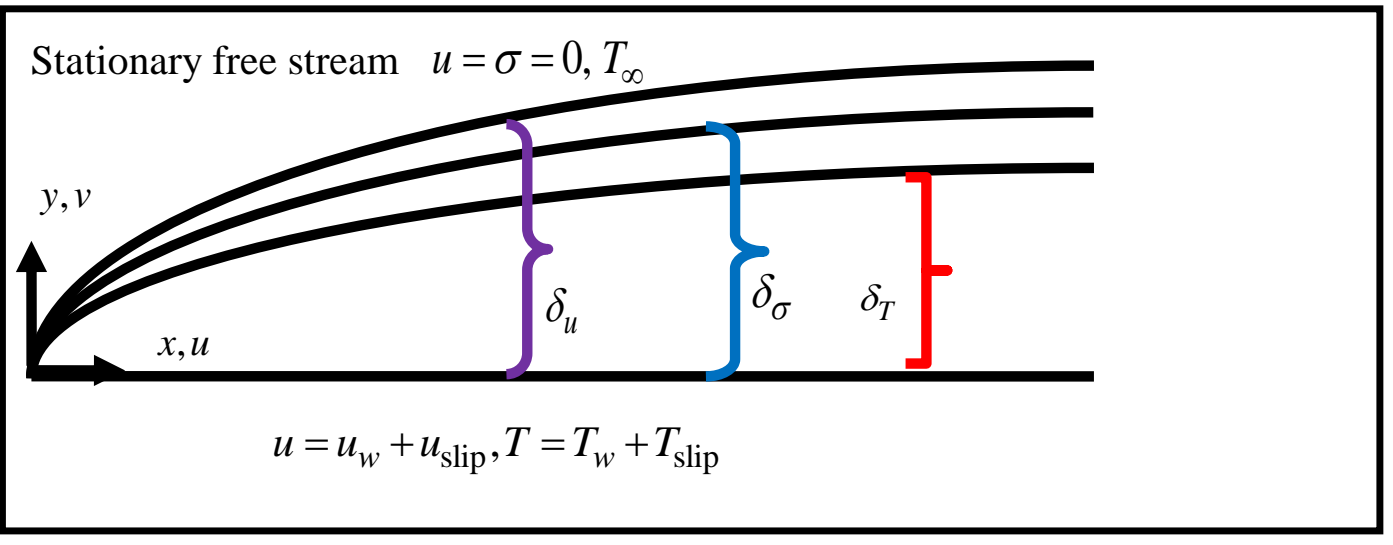

Figure 1: Flow configuration and co-ordinate system

In figure $1, \delta_{u} \delta_{\sigma} \delta_{T}$ represents respectively the velocity, micro-polar and thermal boundary layer thickness. In the $y$-direction a constant magnetic field $B_{0}$ is applied. The viscosity $\mu(T)$ and thermal conductivity $k(T)$ are respectively assumed to be temperature dependent. Under the above assumptions the governing boundary layer equations are assumed as follows: 
$\frac{\partial u}{\partial x}+\frac{\partial v}{\partial y}=0$

$u \frac{\partial u}{\partial x}+v \frac{\partial u}{\partial y}=\frac{1}{\rho_{\infty}} \frac{\partial}{\partial y}\left((\mu(T)+S) \frac{\partial u}{\partial y}\right)+\frac{S}{\rho_{\infty}} \frac{\partial \sigma}{\partial y}-\frac{\sigma_{0} \mathrm{~B}_{0}^{2} \mathrm{u}}{\rho_{\infty}}$

$u \frac{\partial \sigma}{\partial x}+v \frac{\partial \sigma}{\partial y}=\frac{v_{s}}{\rho_{\infty} j} \frac{\partial^{2} \sigma}{\partial y^{2}}-\frac{S}{\rho_{\infty} j}\left(2 \sigma+\frac{\partial u}{\partial y}\right)$

$u \frac{\partial T}{\partial x}+v \frac{\partial T}{\partial y}=\frac{1}{\rho_{\infty} c_{p}} \frac{\partial}{\partial y}\left[k(T) \frac{\partial T}{\partial y}\right]$

The corresponding boundary conditions are:

$\left.\begin{array}{l}\mathrm{y}=0: u=a x+N_{1} \frac{\partial u}{\partial y}, \quad v=0, \quad \sigma=-n \frac{\partial u}{\partial y}, \quad T=T_{w}+\mathrm{E}_{1} \frac{\partial \mathrm{T}}{\partial y}, \\ y \rightarrow \infty^{+} u \rightarrow 0, \quad \sigma \rightarrow 0, \quad T \rightarrow T_{\infty},\end{array}\right\}$

In eqn. (5) the surface parameter $n$ assumes values between and with 0 and 1 that quantifies the relationship between the micro-gyration vector to the shear stress. When $n=0$, this corresponds to the case where the micro-element (particle) density is sufficiently large so that microelements close to the wall are not able to rotate i.e. strong concentration of micro-elements. When $n=0.5$ this indicates weak concentration of micro-elements and the disappearance of the anti-symmetric part of stress tensor, as elaborated by Ahmadi (1976). When $n=1.0$ represents turbulent boundary layer flows as described by Peddieson (1972) and Stokes (1984). Note that in the micro-polar theory vectors are considered to be rigid directors as the micro-elements are non-deformable. The following similarity transformations are used to turn the governing non-linear partial differential equations into non-linear ordinary differential equations:

$$
\eta=y \sqrt{\frac{a}{v_{\infty}}}, \psi=\sqrt{a v_{\infty}} x f(\eta), \sigma=\sqrt{\frac{a^{3}}{v_{\infty}}} x g(\eta), \theta=\frac{T-T_{\infty}}{T_{w}-T_{\infty}}, \mu=\mu_{\infty}\left(\frac{\theta_{r}}{\theta_{r}-\theta}\right),
$$


$k=k_{\infty}\left(1+k_{c} \theta\right)$, where $k_{c}=C_{1}\left(T_{w}-T_{\infty}\right)$

The non-dimensional linear momentum, angular momentum (micro-rotation) and heat conservation governing equations then become:

$$
\begin{aligned}
& \left(\frac{\theta_{r}}{\theta_{r}-\theta}+\Delta\right) f^{\prime \prime \prime}+f f^{\prime \prime}-f^{\prime 2}+\frac{\theta_{r}}{\left(\theta_{r}-\theta\right)^{2}} \theta^{\prime} f^{\prime \prime}+\Delta g^{\prime}-M f^{\prime}=0 \\
& \left(\frac{\theta_{r}}{\theta_{r}-\theta}+\frac{1}{2} \Delta\right) \xi g^{\prime \prime}-\Delta\left(2 g+f^{\prime \prime}\right)-\xi\left(f^{\prime} g-f g^{\prime}\right)=0 \\
& \left(1+k_{c} \theta\right) \theta^{\prime \prime}+k_{c} \theta^{\prime 2}+\operatorname{Pr}_{\infty} f \theta^{\prime}=0
\end{aligned}
$$

Here $\theta_{r}$ is the variable viscosity parameter (negative for liquids and positive for gases),

$\Delta=\frac{S}{\mu_{\infty}}$ is the micropolar parameter, $M=\frac{\sigma_{0} B_{0}^{2}}{\rho_{\infty} a}$ is the magnetic field parameter, $\xi=\frac{j a}{v_{\infty}}$ is the micro-inertia density parameter, $k_{c}=$ thermal conductivity parameter (for air $0 \leq k_{c} \leq 6$, for water $0 \leq k_{c} \leq 0.12$ and for lubrication oils $\left.-0.1 \leq k_{c} \leq 0\right)$, $\operatorname{Pr}_{\infty}=\frac{\mu_{\infty} c_{p}}{k_{\infty}}$ is the Prandtl number.

The appropriate transformed boundary conditions are:

$$
\begin{aligned}
& f^{\prime}(0)=1+l f^{\prime \prime}(0), f(0)=0, \quad \theta(0)=1+d \theta^{\prime}(0), g(0)=-n f^{\prime \prime}(0), \\
& \eta \rightarrow \infty^{+}: f^{\prime}=0, g=0, \theta=0
\end{aligned}
$$

where $l$ denotes velocity slip parameter and $d$ designates thermal slip parameter. 
The equation defining the surface shear stress at the plate is: $\tau_{w}=(\mu+S)\left(\frac{\partial u}{\partial y}\right)_{y=0}+S(\sigma)_{y=0}$

The dimensionless local skin-friction coefficient is defined as:

$$
C f_{x}=\frac{\tau_{w}}{\frac{1}{2} \rho_{\infty} u_{w}^{2}}=\frac{2 \tau_{w}}{\rho_{\infty}(a x)^{2}}=\frac{2}{\sqrt{\operatorname{Re}_{x}}}\left[\frac{\theta_{r}}{\theta_{r}-\theta}+\Delta(1-n)\right] f^{\prime \prime}(0)
$$

The equation defining the local wall couple stress (micro-rotation gradient) is:

$$
M_{w}=v_{s}\left(\frac{\partial \sigma}{\partial y}\right)_{y=0}=\left(\mu+\frac{S}{2}\right) j\left(\frac{\partial \sigma}{\partial y}\right)_{y=0}
$$

The dimensionless couple stress is defined by:

$M_{x}=\frac{2 M_{w}}{(\mu+S)(a x)}=\frac{\left(\frac{2 \theta_{r}}{\theta_{r}-\theta}+\Delta\right) \xi}{\frac{\theta_{r}}{\theta_{r}-\theta}+\Delta} g^{\prime}(0)$

The local surface heat flux is expressed as:

$q_{w}(x)=-k(T)\left(\frac{\partial T}{\partial y}\right)_{y=0}=-k_{\infty}\left(1+k_{c} \theta\right) \theta^{\prime}(0) \sqrt{\frac{a}{v_{\infty}}}$

The heat transfer coefficient is:

$h(x)=\frac{q_{w}(x)}{T_{w}-T_{\infty}}=-k_{\infty}\left(1+k_{c} \theta\right) \theta^{\prime}(0) \sqrt{\frac{a}{v_{\infty}}}$

The local Nusselt number is:

$$
N u_{x}=\frac{x h(x)}{k(T)}=-\sqrt{\operatorname{Re}_{x}} \theta^{\prime}(0)
$$

\section{NUMERICAL COMPUTATION AND VALIDATION}

An initial value solver shooting method termed the Nachtsheim-Swigert (1965) iteration technique together with the sixth order Runge-Kutta-Butcher iteration scheme has been 
used to solve the coupled nonlinear differential Eqns. (7)-(9) subject to the boundary conditions (10a)-(10b). A step size $\Delta \eta=0.001$ has been chosen to satisfy the convergence criterion of $10^{-6}$ in all cases. The maximum value of $\eta_{\infty}=\eta_{\infty}+\Delta \eta$ is ascribed to each group of parameters $\theta_{r}, \Delta, M, \xi, k_{c}, \operatorname{Pr}_{\infty}$ and $n$ when the value of the unknown boundary conditions at $\eta=0$ change, to a successful loop with error less than $10^{-6}$. To verify the shooting computations, we have compared the numerical solutions for local skin-friction coefficient $f^{\prime \prime}(0)$ with those of Hamad et al. (2012) by considering $M=0$ and $\theta_{r} \rightarrow \infty$ (i.e. non-magnetic constant thermal conductivity scenario) for different values of slip parameter $l$. From Table $\mathbf{1}$ it is evident that very good correlation is obtained and confidence in the shooting code is therefore high.

Table 1: Comparison of $f^{\prime \prime}(0)$ for various values of $l$.

\begin{tabular}{|c|c|c|c|}
\hline Slip parameter $l$ & Hamad et al. (2012) & Shooting Quadrature & ADM \\
\hline 0.0 & 1.0000 & 1.0000 & 1.0000 \\
\hline 0.1 & 0.9554 & 0.9533 & 0.9549 \\
\hline 0.2 & 0.7763 & 0.7602 & 0.7738 \\
\hline
\end{tabular}

\section{VALIDATION WITH ADOMIAN DECOMPOSITION METHOD (ADM)}

To justify the correctness of the present model with all parameters invoked, an alternative numerical method is required. We used the efficient Adomian decomposition method (ADM) which is a semi-numerical technique utilizing special Adomian polynomials to achieve very accurate solutions which may be evaluated using symbolic packages such as Mathematica. An advantage of this method is that it can provide analytical approximation or an approximated solution to a wide class of nonlinear equations without linearization, perturbation closure approximation or discretization methods. ADM has gained popularity in modern engineering sciences and has been used for simulation of Newtonian flows (Liu, 2016), Sisko non-Newtonian thin film flows (Siddiqui et al., 
2013), bio-magnetic orthopaedic lubrication flows (Bég et al., 2015), micropolar channel hydrodynamics in channels (Aski et al., 2014), entropy minimization in micropolar flow (Jangili et al., 2017), pulsatile micropolar flow (Adanhounmè et al., 2012) and stagnation-point rotating nanofluid flows (Bég et al., 2015). ADM (Adomian, 1994) deploys an infinite series solution for the unknown functions and utilizes recursive relations. For example for the $7^{\text {th }}$ order nonlinear ordinary differential Eqns. (7)-(9), we assume infinite series solution for the unknown linear velocity, micro-rotation, and temperature functions $f(\eta), h(\eta), \theta(\eta)$, defined as follows:

$$
\begin{aligned}
& f(\eta)=\sum_{n=0}^{\infty} f_{n}(\eta) \\
& h(\eta)=\sum_{n=0}^{\infty} h_{n}(\eta) \\
& \theta(\eta)=\sum_{n=0}^{\infty} \theta_{n}(\eta)
\end{aligned}
$$

The components $f_{0}, f_{1}, f_{2} \ldots . ., h_{0}, h_{1}, h_{2} \ldots .$. , and $\theta_{0}, \theta_{1}, \theta_{2} \ldots$. are usually obtained recursively by an appropriate relation, as elaborated further by Bég et al. (2015). The resulting decomposition series converges very quickly and relatively few terms are needed to achieve high accuracy. Comparison between shooting and ADM solutions for selected values of certain parameters are shown in Figs $3 b$ (temperature), 4a (velocity) and $6 \mathrm{~b}$ (couple stress). For all cases very close correlation is found. Furthermore, we have verified the ADM solution against the results of Hamad et al. (2012) in Table 1. Confidence in the shooting solutions is therefore again justifiably high.

\section{RESULTS AND DISCUSSION}

Selected shooting method solutions for linear velocity, temperature, local skin-friction coefficient, local wall couple stress and local Nusselt number have been visualized in Figs 2-6. 
Figs. 2 (a), (b) respectively, illustrate the velocity and temperature profiles for various magnetic field parameter values $(M)$ for both cases of strong $(n=0)$ and weak $(n=0.5)$ micro-element concentration. It can be seen from Fig. 2 (a) that velocity profiles decrease with the increase of magnetic field parameter $M$. Increasing $M$ implies a stronger applied magnetic field. This enhances the Lorentz magnetohydrodynamic drag force which inhibits the micropolar flow.
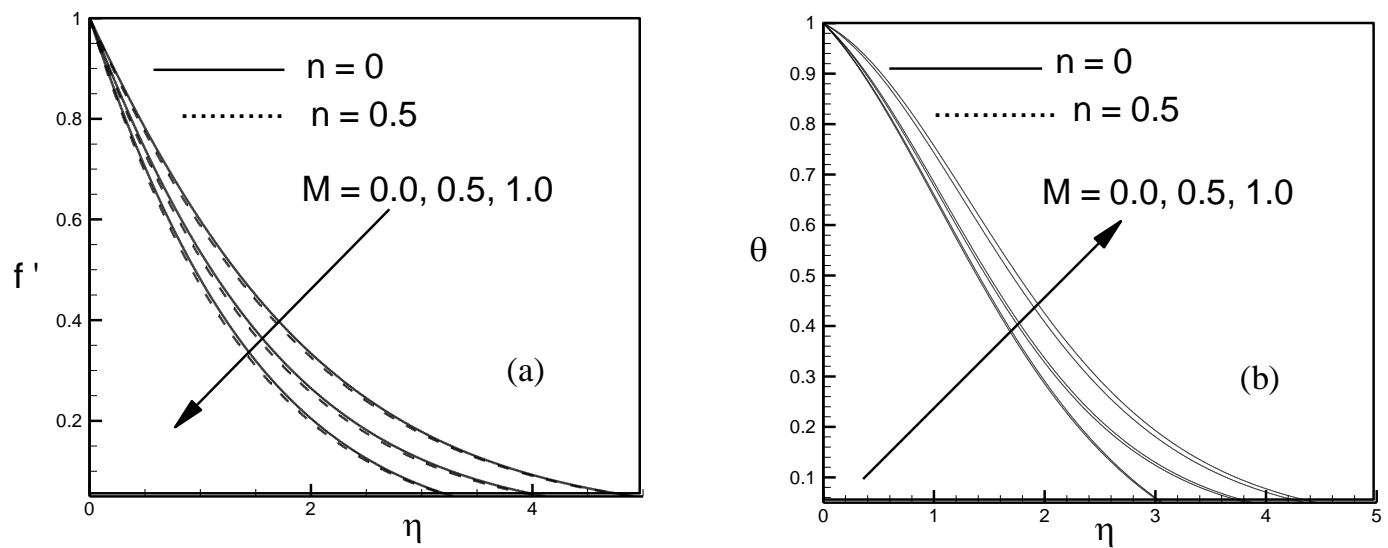

Fig. 2: Magnetic field parameter $(M)$ effects on (a) velocity and (b) temperature profiles. 

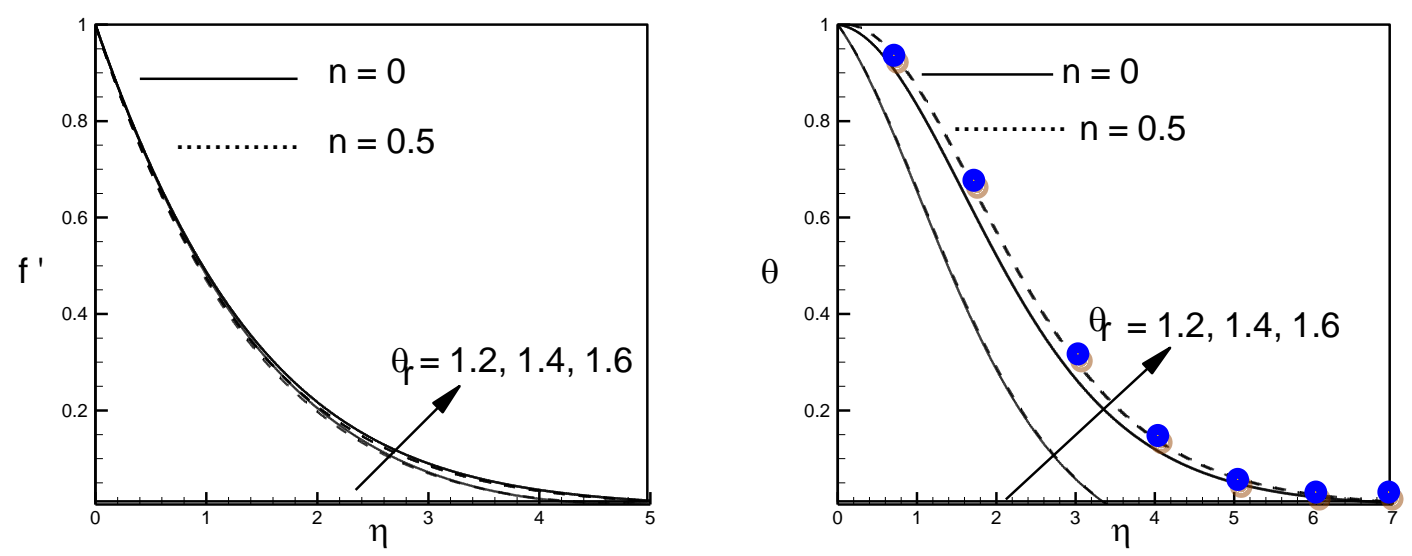

Fig. 3: Variable viscosity parameter $\left(\theta_{r}\right)$ effects on (a) velocity and (b) temperature profiles.[Blue dots are ADM solution]
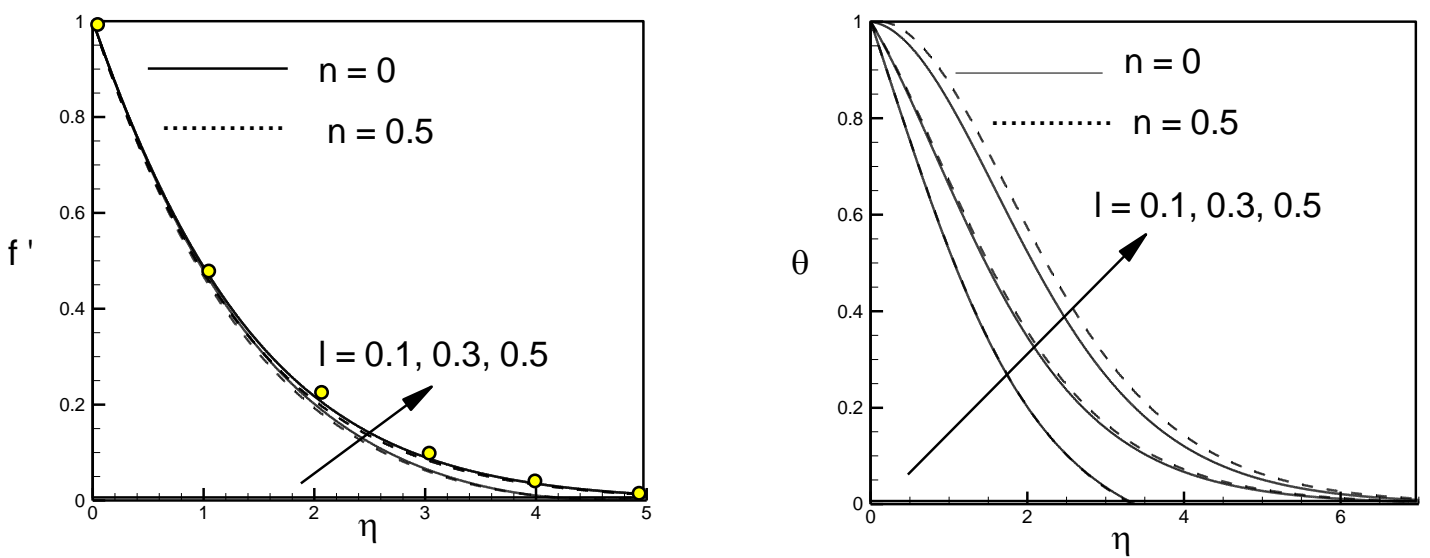

Fig. 4: Velocity slip parameter $(l)$ effect on (a) velocity and (b) temperature profiles. [Yellow dots are ADM solution] 

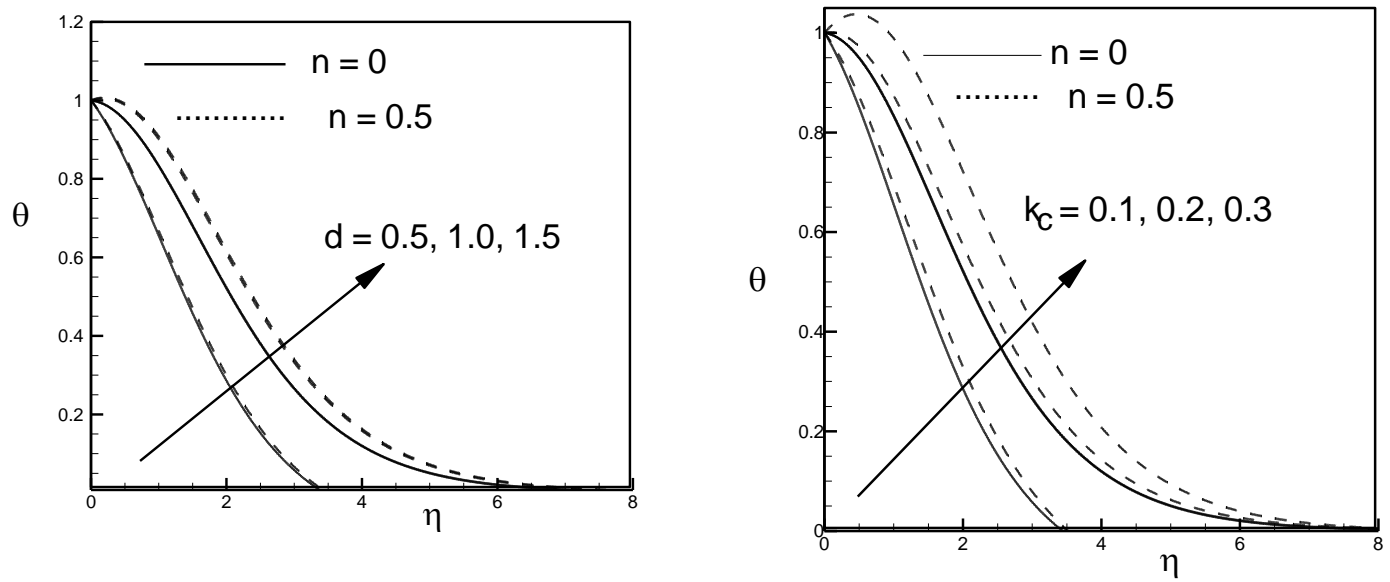

Fig. 5: (a) Thermal slip parameter $(d)$ and (b) thermal conductivity parameter $\left(k_{c}\right)$ effects on temperature profiles.

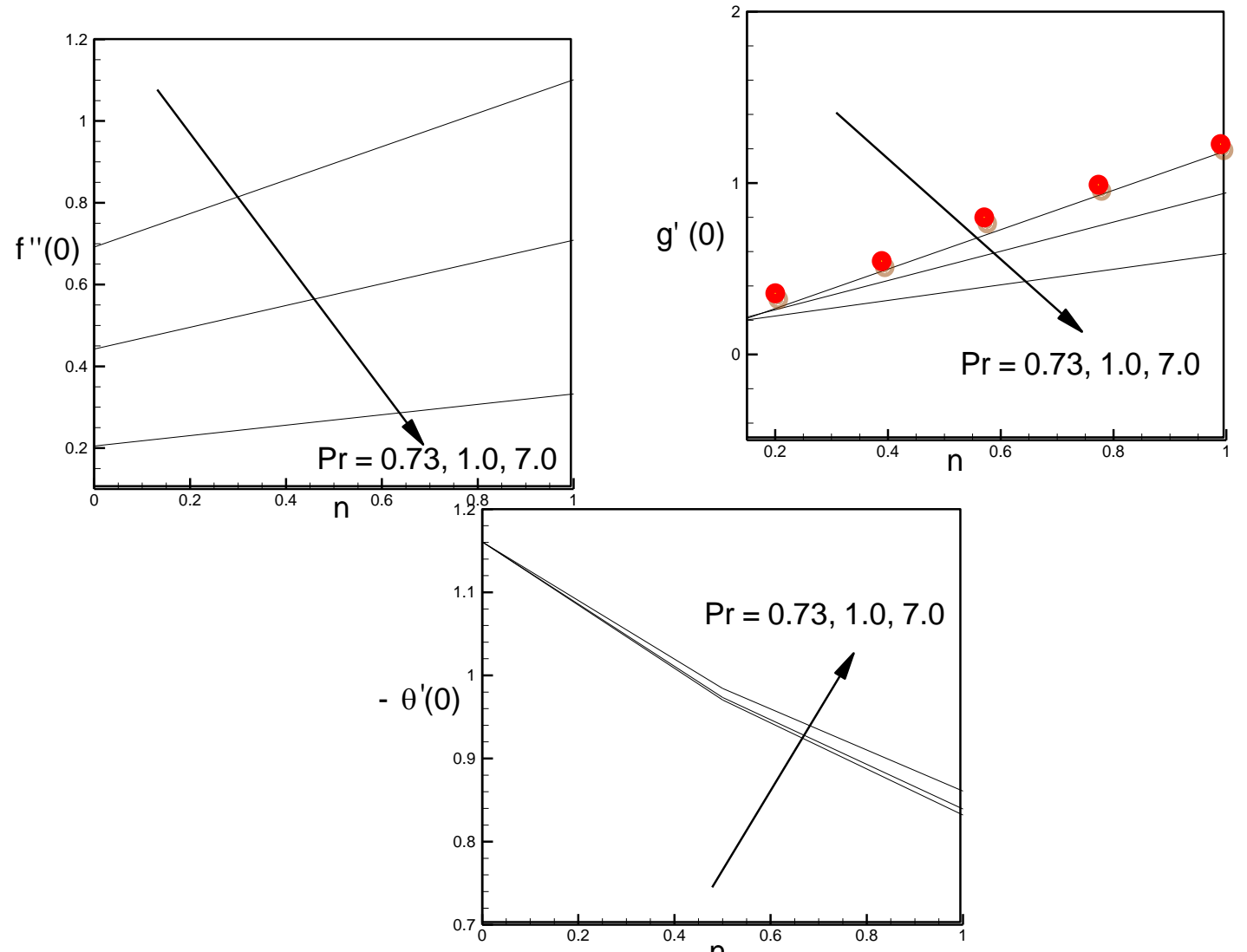

Fig. 6: (a) Local skin-friction coefficient $\left(C f_{x}\right)$, (b) local plate couple stress $\left(M_{x}\right)$ and (c) local Nusselt number $\left(N u_{x}\right)$ profiles for different values of $n$ and $\operatorname{Pr}_{\infty}$.[Red dots are ADM solution] 
In consistency with numerous other studies, notably Sutton and Sherman (1965), Rossow (1958), Sarpkaya (1961) and Greenspan and Carrier (1959), with greater $M$ values the Lorentz magnetic drag force is elevated. This retards the momentum development and decelerates the boundary layer flow. Hydrodynamic boundary layer thickness is therefore increased. Greater concentration $(n=0)$ of micro-elements at the plate surface results in marginally greater velocity than for the case of weak concentration $(n=0.5)$. This may be attributable to the surface enhancement in linear momentum caused by reduced angular momentum of the rotating micro-elements via coupling in the momenta eqns. (7) and (8). The de-intensification in gyratory motions with stronger concentrations (micro-elements are stifled in gyratory motions) acts as a source boosting the linear momentum, via momentum re-distribution. A similar observation has been noted by Gorla et al. (1990). The case of $M=0$ correspond to vanishing magnetic field i.e. the micropolar fluid loses electrical conductivity properties. The Lorentz drag force is therefore eliminated and the velocity is a maximum in this case i.e. hydrodynamic boundary layer thickness is a minimum. From Fig. 2 (b) we observe that temperature profiles increase with the increase of magnetic field parameter $M$ and this increasing effect is amplified for the case of weak concentration of micro-elements $(n=0.5)$ relative to that of strong concentration $(n=0)$. The supplementary work expended by the magnetized micropolar fluid in dragging against the action of the magnetic field is dissipated as thermal energy i.e. heat. This elevates temperatures and increases thermal boundary layer thickness. In consistency with this the minimum temperature generated corresponds to $M=0$ (electrically nonconducting case). It is noteworthy that based on the definition of magnetic body force parameter, $M=\frac{\sigma_{0} B_{0}^{2}}{\rho_{\infty} a}$, for $M=1$ there is an equivalence in magnetic drag and inertial forces in the regime. For $M<1$ the inertial force dominates the magnetic force.

Figs. 3 (a) and (b) show the variation of velocity and temperature profiles respectively with variable viscosity parameter, $\theta_{r}$. Fig. 3 (a) shows that the velocity increases significantly further from the plate surface as the variable viscosity parameter $\theta_{r}$ is 
increased. $\theta_{r}=\left(\frac{\mu}{\mu-\mu_{\infty}}\right) \theta$ and clearly increasing values imply that the zero shear rate viscosity is decreased. The viscous drag force at the plate is therefore reduced and this accelerates the flow which simultaneously reduces momentum boundary layer thickness. The temperature profiles as well as thermal boundary layer increase as the variable viscosity parameter $\theta_{r}$ increases. At lower values of viscosity parameter (i.e. 1.2) the temperature does not descend smoothly to zero; it intersects the abscissa much earlier than at higher values of $\theta_{r}$. This behavior is not associated with the infinity boundary condition i.e. it is not a numerical anomaly. The trend is eliminated with higher values of $\theta_{r}$ where smooth decay is achieved in the free stream. The temperature distribution is therefore very sensitive to viscosity variation. This has also been confirmed by Nield and Kuznetsov (2003).

Figs. 4 (a) and (b) illustrate the evolution in velocity and temperature profiles with an alteration in hydrodynamic slip parameter $(l)$. There is a distinct accentuation in velocity profiles increase further from the plate; the flow is therefore accelerated with increasing hydrodynamic slippage at the plate and this effect is carried quite deeply into the boundary layer, transverse to the plate. Physically this may correspond to molecular nonadherence of the micropolar fluid to the wall, which encourages boundary layer growth. This pushes the interfacial region in the growth direction and feeds momentum leading to a reduced momentum boundary layer thickness. The no-slip model $(l=0)$ inevitably under-predicts the wall skin friction effect. Similar observations have been made by Stewart et al. (1993). The macroscopic weak slip can be modelled with a first order isotropic slip factor (as in the current model) which is strongly connected to flow-induced chain detachment/desorption at the polymer/wall interface. Micropolar fluids may therefore offer a good approximation for interfacial wall dynamics of polymers in addition to complex non-Newtonian suspensions. However the first slip isotropic model employed is still inadequate to capture the stronger slip effect which is associated with chain disentanglement of for example polymer chains in the bulk from a monolayer of polymer chains adsorbed at the interface. This more complex slip (which may also be 
anisotropic) is currently under investigation and has infact been considered by the authors in an earlier non-magnetic study (Uddin et al. 2016). Temperature profile increases strongly with stronger velocity slip parameter $l$. The temperature magnitudes are also greater for the case of weak micro-element concentration compared with strong concentration. Thermal boundary layer thickness is clearly enhanced with greater momentum wall slip effect. It is likely that the greater momentum diffusion (linear) via coupling with the convective term in the energy equation (9) i.e. $P r_{\circ} f \theta$ influences the temperature distribution also. There is also strong coupling via the fourth term on the left hand side of the momentum eqn. (7). Thermal diffusion in the boundary layer regime is probably assisted quite markedly with wall slip which also encourages heat transfer away from the plate surface into the micropolar fluid. This energizes the boundary layer.

Figs. 5 (a) and (b) respectively show the effects of thermal slip parameter $d$ and thermal conductivity parameter $k_{c}$ on temperature profiles. Temperature values and consequently thermal boundary layer thickness are boosted with higher thermal slip and also thermal conductivity parameter $k_{c}$. Thermal jump at the wall introduces heat into the boundary layer. It assists thermal conduction as well as thermal convection currents. The heat is dispersed strongly into the boundary layer. This will cool the wall progressively and encourage thermal diffusion in the micropolar fluid. Similarly an increase in thermal conductivity decreases the thermal resistance of the regime (they are inversely related). This allows thermal energy to move more efficiently and quickly through the micropolar fluid and enhances the conveyance of heat throughout the boundary layer. Faster cooling of the wall is therefore achieved with greater thermal conductivity of the micropolar fluid. Similar findings have been documented by Zueco et al (2011). It is also evident from the figures that temperature profiles overshoot for higher values of $d$ and $k_{c}$ and larger magnitudes are achieved with weak micro-element concentration at the wall (n $=0.5)$.

Figs. 6(a)-(c), respectively, show the response in the local skin-friction factor, local wall couple stress and local Nusselt number for different values of micro-rotation surface parameter $(n)$ and Prandtl number $(\operatorname{Pr})$. There is a considerable growth in the skin- 
friction, and wall couple stress (micro-rotation i.e. angular velocity gradient) with an increase in micro-rotation parameter $n$. Conversely there is a reduction in Nusselt number. The gyratory motions of the micro-elements at the wall influence both velocity and angular velocity significantly. When less micro-elements are present (weak concentration i.e. higher $n$ value), they are able to rotate freely and enhanced the angular momentum. This is not possible at strong concentration $(n=0)$. This via coupling in the conservation similarity eqns. $(7,8)$ leads to greater linear momentum also in the regime. The micropolar fluid therefore shears faster against the plate and achieves higher couple stress in this vicinity. The figures also indicate that skin-friction coefficient and wall (plate) couple stress are reduced whereas the Nusselt number (heat transfer gradient at the plate) is increased with a rise in Prandtl number, $\operatorname{Pr}$. An increase in $\operatorname{Pr}$ from 0.73 (air, gas) through 1 to 7 (water-based suspensions, low-density polymeric aqueous solutions) substantially decelerates the flow i.e. reduces hydrodynamic boundary layer thickness. This results in a decrease in skin friction. The temperature also decays quickly for large values of Prandtl number. The thermal boundary layer thickness is similarly the distance from the body at which the temperature is $99 \%$ of the temperature found from an inviscid solution. The ratio of the two thicknesses is dictated by the Prandtl number. For Prandtl number of unity, both boundary layers are of the same thickness i.e. order of magnitude. However when Prandtl number exceeds unity, the thermal boundary layer is thinner than the velocity boundary layer. Generally, higher $\operatorname{Pr}$ fluids will have relatively low thermal conductivities which will suppress thermal conduction heat transfer from the wall and reduce thermal boundary layer thickness, resulting in lower micropolar fluid temperatures in the boundary layer regime. Thermal energy will be transported to the plate (wall) and this results in the computed elevation in Nusselt number. Smaller values of $\operatorname{Pr}$ are equivalent to increasing thermal conductivities, and therefore heat is able to diffuse away from the heated plate more rapidly than for higher values of $\mathrm{Pr}$. Hence in the case of smaller $\operatorname{Pr}$ the boundary layer is thicker and the rate of heat transfer to the wall i.e. Nusset number is reduced. This has important implications in materials processing since by changing the Prandtl number (related to thermo-physical properties of the micropolar liquid) the heat transfer characteristics can be dramatically modified. Faster cooling is achieved with denser micropolar liquids compared with lighter ones. 


\section{CONCLUSIONS}

Computational solutions have been presented for variable viscosity and variable thermal conductivity magnetized steady-state incompressible micropolar heat transfer from a plate. Both momentum and thermal slip effects have been included. Runge-Kutta numerical quadrature has been employed to solve the transformed ordinary differential boundary value problem. Verification of solutions with earlier non-magnetic non slip studies has also been conducted. General validation of the model developed has also been included using an Adomian decomposition method (ADM). In the present work significant attention has also been addressed to the surface micro-rotation effect which is simulated by a boundary condition. The current study has shown that:

- Magnetic field parameter can be used to control the flow characteristics and temperature fields in micropolar fluids. Stronger transverse magnetic field decelerates the flow and elevates the temperature.

- Increasing viscosity parameter and hydrodynamic (momentum) slip accelerate the flow further from the plate surface.

- The thermal boundary layer thickness is noticeably enhanced with greater viscosity parameter.

- Temperature and thermal boundary layer thickness are both elevated markedly with an increase in velocity slip, thermal slip parameter and thermal conductivity parameter. These effects are more pronounced for the case of weak concentration of micro-elements at the plate surface.

- Skin-friction coefficient and wall couple stress are both increased whereas Nusselt number is reduced with an elevation in micro-rotation surface parameter.

- Skin-friction coefficient and wall couple stress are reduced whereas Nusselt number is boosted strongly (i.e. temperature and thermal boundary layer thickness are decreased) with an increase in Prandtl number.

The current study has considered a flat pate geometrical configuration and has ignored curvature effects. These will be addressed in future studies. Additionally, anisotropic slips will also be considered. 


\section{REFERENCES}

Adanhounmè, V.F., Codo, D.P., Adomou, A., 2012. Solving the Navier Stokes flow equations of micro-polar fluids by Adomian decomposition method, Bull. Math. Sc. Appl. 2, 30-37.

Adomian, G., 1994. Solving Frontier Problems in Physics: The Decomposition Method, Kluwer, Dordrecht, USA.

Ahmadi, G., 1976. Self-similar solution of incompressible micropolar boundary layer flow over a semi-infinite plate. Int. J. Eng. Sci. 14, 639-646.

Akbar, N.S., Tripathi, D., Bég, O. Anwar, Khan, Z.H., 2016. MHD dissipative flow and heat transfer of Casson fluids due to metachronal wave propulsion of beating cilia with thermal and velocity slip effects under an oblique magnetic field, Acta Astronautica, 128, $1-12$.

Akyuzlu, K.M., 2015. Numerical study of high-temperature and high-velocity gaseous hydrogen flow in a cooling channel of a nuclear thermal rocket core, ASME J. Nuc. Rad. Sci. 1(4):041006-041006-13.

Ariman, T., Turk, M.A. and Sylvester N. D., 1974. Micro-continuum fluid mechanics-a review, Int. J. Eng. Sci. 11, 905-930.

Ashraf, M. and Batool, K., 2013. MHD flow and heat transfer of a micropolar fluid over a stretchable disk, J. Theo. Appl. Mec. 51, 25-38.

Aski, F.S., Nasirkhani, S.J., Mohammadian, E. and Asgari, A., 2014. Application of Adomian decomposition method for micropolar flow in a porous channel, Propulsion and Power Research, 3, 15-21.

Bég, O. Anwar, Zueco, J. and Chang, T.B., 2010. Numerical analysis of hydromagnetic gravity-driven thin film micropolar flow along an inclined plane, Chem. Eng. Comm. 198, 3, 312-331.

Bég, O. Anwar, Zueco, J. and Ghosh, S.K., 2010. Unsteady natural convection of a shortmemory viscoelastic fluid in a non-Darcian regime: Network simulation, Chem. Eng. Comm. 198, 172-190.

Bég, O. Anwar, Zueco, J. and López-Ochoa, L.M., 2011. Network numerical analysis of optically-thick hydromagnetic slip flow from a porous spinning disk with radiation flux, variable thermophysical properties and surface injection effects, Chem. Eng. Comm. 198, 3, 360-384. 
Bég, O. Anwar, Rashidi, M.M., Bég, T.A. and Asadi, M., 2012. Homotopy analysis of transient magneto-bio-fluid dynamics of micropolar squeeze film: a model for magnetobio-rheological lubrication, J. Mec. Med. and Bio. 12, 1250051-1 to 1250051-21.

Bég, O. Anwar, Tripathi, D., Sochi, T. and Gupta, P.K., 2015. Adomian decomposition method (ADM) simulation of magneto-bio-tribological squeeze film with magnetic induction effects, J. Mec. Med. Bio. 15, 1550072.1-1550072.23.

Bég, O. Anwar, Mabood, F. and Islam, M.N. 2015. Homotopy simulation of nonlinear unsteady rotating nanofluid flow from a spinning body, Int. J. Eng. Math. Volume 2015, Article ID 272079, 15 pages.

Bég, O.Anwar, Motsa, S.S., Kadir, A., Bég, T.A. and Islam, M.N., 2016. Spectral quasilinear numerical simulation of micropolar convective wall plumes in high permeability porous media, J. Eng. Therm. 25 (4) 1-24.

Bhatti, M.M., Shahid, A., Bég, O. Anwar and Kadir, A., 2017. Numerical study of radiative Maxwell viscoelastic magnetized flow from a stretching permeable sheet with the Cattaneo-Christov heat flux model, Neu. Comput. Appl. DOI 10.1007/s00521-0172933-8 (12 pages)

Bogdanova, E.V., Zotov, V.G., Malkin, S.A., Vitkovskii, I.V., Kirillov, I.R., Kornov, K.A. and Federyaeva, V.S., 2017. Possibility and desirability of using electromagnetic pumps in the emergency heat removal systems of fast reactors, Atomic Energ. 122, 178184.

Corradini, M.L., 2003. Advanced nuclear energy systems: heat transfer issues and trends, Rohsenow Symposium on Future Trends in Heat Transfer: MIT, May $16^{\text {th }}$, USA.

Doležel, I., Donátová, M., Karban, P., Ulrych, B., 2009. Pumps of molten metal based on magnetohydrodynamic principle for cooling high-temperature nuclear reactors, Przeglad Elektrotechniczny (Electrical Review), 4, 13-16.

Eringen, A.C., 1964. Simple micro fluids, Int. J. Eng. Sci. 2, 205-217.

Eringen, A.C., 1966. Theory of micropolar fluids, J. Math. Mech. 16, 1-18.

Eringen, A.C., 2001. Microcontinuum field theories II: fluent media, Springer, New York. 1-80.

Gorla, R.S.R., Lin, P.P., Yang, A., 1990. Asymptotic boundary layer solutions for mixed convection form a vertical surface in a micropolar fluids. Int. J. Eng. Sci. 28, 525-533.

Gradecka, M., Thiele, R. and Anglart, H., 2016. Computational fluid dynamics investigation of supercritical water flow and heat transfer in a rod bundle with grid spacers, ASME J. Nuclear Rad. Sci. 2(3):031015-031015-9. 
Greenspan, H.P. and Carrier, G.F., 1959. The time-dependent magnetohydrodynamic flow past a flat plate, J. Fluid Mech. 6, 228.

Gupta, D., Kumar, L., Bég, O.Anwar and Singh, B., 2015. Finite element simulation of nonlinear magneto-micropolar stagnation point flow from a porous stretching sheet with prescribed skin friction, Comput. Therm. Sci. 7 (1): 1-14.

Hamad, M.A.A., Uddin, M.J., Ismail, A.I.M., 2012. Investigation of combined heat and mass transfer by Lie group analysis with variable diffusivity taking into account hydrodynamic slip and thermal convective boundary conditions, Int. J. Heat and Mass Trans. 55, 1355-1362.

Harvego, E.A., Mckellar, M.G., Sohal, M.S. and Herring, J.S., 2010. System evaluation and economic analysis of a nuclear reactor powered high-temperature electrolysis hydrogen-production plant, ASME J. Energy Resour. Technol., 132(2):021005-021005-9.

Hayat, T., Naz, R., Asghar, S. and Mesloub, S., 2012. Soret-Dufour effects on threedimensional flow of third grade fluid, Nuclear Eng. Design, 243, 1-14.

Huda, A.B., Akbar, N.S., Bég, O. Anwar and Khan, M.Y., 2017. Dynamics of variableviscosity nanofluid flow with heat transfer in a flexible vertical tube under propagating waves, Res. Phys. 7, 413-425

Ishak, A., Nazar, R. and Pop, I., 2006. Flow of a micropolar fluid on a continuous moving surface, Arch. Mech. 58, 529-541.

Ismail, S., Sarangi, M., 2013. Effects of texture shape and fluid-solid interfacial slip on the hydrodynamic lubrication performance of parallel sliding contacts, Proceedings of the Institution of Mechanical Engineers, Part J: J. Eng. Tribol. 228, 382-396.

Jangili, S., Gajjela, N., and Bég, O. Anwar, 2016. Mathematical modelling of entropy generation in magnetized micropolar flow between co-rotating cylinders with internal heat generation, Alexandria Eng. J. 55(3), 1969-1982.

Jangili, S., Adesanya, S.O., Falade, J.A., Gajjela, N., 2017. Entropy generation analysis for a radiative micropolar fluid flow through a vertical channel saturated with nonDarcian porous medium, Int. J. Appl. Comp. Math, 3(4), 3759-3782)

Jena, S.K. and Mathur, M.N., 1981. Similarity solution for laminar free convection flow of thermomicropolar fluid past a non-isothermal vertical flat plate, Int. J. Eng. Sc. 19, 1431-1439.

Khader, M.M., Megahed, A.M., 2014. Differential transformation method for the flow and heat transfer due to a permeable stretching surface embedded in a porous medium 
with a second order slip and viscous dissipation, ASME J. Heat Trans. 136(7):072602072602-7.

Khedr, M.E.M., Chamkha, A.J. and Bayomi, M., 2009. MHD flow of a micropolar fluid past a stretched permeable surface with heat generation or absorption, Nonlinear Anal. Model. Control, 14 (1), 27-40.

Kim, S.H., Kim, I.S. and Kim, J.H., 2005. Effect of a magnetic field on the flow assisted corrosion of low alloy steel in an alkaline solution, J. Nuclear Sci. Technol. 42, 559-565.

Kirillov, P.L., 2016. Colligation of experimental data in terms of transfer in liquid metals, Atomic. Énerg. 120, 198-201.

Kumar, T.S, Kumar, B.R, Makinde, O.D., Vijaya Kumar, A.G, 2018, MagnetoConvective Heat Transfer in Micropolar Nanofluid over a Stretching Sheet with NonUniform Heat Source/Sink. Defect and Diffusion Forum, 387, 78-90.

Latiff, M.A.A., Uddin, M.J., Bég, O. Anwar and Ismail, A.I.M., 2015. Unsteady forced bioconvection slip flow of a micropolar nanofluid from a stretching/shrinking sheet, Proceedings of the Institution of Mechanical Engineers, Part N: J. Nanomat. Nanoeng. Nanosys. 230, 177-187.

Lahjomri, J. and Oubarra, A., 2013. Hydrodynamic and thermal characteristics of laminar slip flow over a horizontal isothermal flat plate, ASME J. Heat Trans. 135(2):021704021704-9 (2013).

Lai, F.C. and Kulacki, F.A., 1990. The effect of variable viscosity on convective heat and mass transfer along a vertical surface in saturated porous media, Int. J. Heat Mass Trans. $33,1028-1031$.

Liu, C.M., 2016. Application of the Adomian Decomposition Method to oscillating viscous flows, Appl. Comput. Math. 5, 121-132.

Modather, M., Abdou, M. and Roshdy El-Zahar E., 2012. Variable viscosity effect on heat transfer over a continuous moving surface with variable internal heat generation in micropolar fluids, Appl. Math. Sci, 6, 128, 6365-6379.

Mahanthesh, B., Gireesha, B.J, Gorla, R.S.R., Makinde, O. D., 2018, Magnetohydrodynamic three-dimensional flow of nanofluids with slip and thermal radiation over a nonlinear stretching sheet: a numerical study, Neural Computing and Appli., 30(5), 1557-1567.

Mukhopadhyay, S., 2011. Heat transfer analysis for unsteady MHD flow past a nonisothermal stretching surface, Nucl. Eng. Des. 241, 4835-4839. 
Mukhopadhyay, S., 2011. Effects of slip on unsteady mixed convective flow and heat transfer past a porous stretching surface, Nucl. Eng. Des. 241, 2660-2665.

Nachtsheim, P.R. and Swigert, P., 1965. Satisfaction of the asymptotic boundary conditions in numerical solution of the system of nonlinear equations of boundary layer type, NASA TND-3004, USA.

Naveed, M., Abbas, Z. and Sajid, M., 2016. MHD Flow of micropolar fluid due to a curved stretching sheet with thermal radiation, J. Appl. Fluid Mech. 9(1), 131-138.

Nield, D.A. and Kuznetsov, A.V., 2003. Effects of temperature-dependent viscosity on forced convection in a porous medium: layered-medium analysis, J. Porous Media, 6:213-222.

Peddieson, J., 1972. Boundary-layer theory for a micropolar fluid. Int. J. Eng. Sci.10, 23-29.

Prasad, V.R., Rao, A.S., Reddy, N.B., Vasu, B. and Bég, O. Anwar, 2012. Modelling laminar transport phenomena in a Casson rheological fluid from a horizontal circular cylinder with partial slip, Proceedings of the Institution of Mechanical Engineers, Part E: J. Process Mech. Eng. 227, 309-326.

Rahman, M.M., Al-Rashdi, Maryam, H. and Pop, I., 2016. Convective boundary layer flow and heat transfer in a nanofluid in the presence of second order slip, constant heat flux and zero nanoparticles flux, Nucl. Eng. Des. 297, 95-103.

Reddy, K.S.N., Babu, M.S., Varma, S.V.K. and Reddy, N.B., 2014. MHD stagnation point flow of a micropolar fluid over a stretching surface with heat source/sink, chemical reaction and viscous dissipation, Int. J. Eng. Inven. 3(10), pp.15-26.

Rees, D.A.S. and Bassom, A.P., 1996. The Blasius boundary-layer flow of a micropolar fluid, Int. J. Eng. Sci. 34, 113-124.

Rossow, V.J., 1958. On flow of electrically conducting fluid over a flat plate in the presence of a transverse magnetic field, NASA Report No. 1358, USA.

Sarpkaya, T., 1961. Flow of non-Newtonian fluids in a magnetic field, AIChE. J., 7, 324328.

Sarojamma, G., Lakshmi, R.V., Narayana, P.V.S., Makinde, O.D., 2018, Non Linear Radiative Flow of a Micropolar Nanofluid through a Vertical Channel with Porous Collapsible Walls. Defect and Diffusion Forum, 387, 498-509, 2018.

Satapathy, A.K., 2010. Slip flow heat transfer in a semi-infinite microchannel with axial conduction, Proceedings of the Institution of Mechanical Engineers, Part C: J. Mech. Eng. Sci. 224, 357-361. 
Schowalter, W.R., 1978. Mechanics of Non-Newtonian Fluids, Pergamon Press, OxfordFrankfurt.

Siddiqui, A.M., Ashraf, H., Haroon, T. and Walait, A., 2013. Analytic solution for the drainage of Sisko fluid film down a vertical belt, Appl. Appl. Math. 8, 465-470.

Stewart, C.W., McMinn, R.S. and Stika, K.M., 1993. A model for predicting slip velocity during extrusion with fluoropolymer processing additives, J. Reinf. Plast. Compos. 12, 633-641.

Stokes, V.K., 1984. Theories of Fluids with Microstructure, Springer, New York.

Sutton, G.W. and Sherman, A., 1965. Engineering Magnetohydrodynamics, McGrawHill, New York, USA.

Thakur, P.M. and Hazarika, G.C., 2015. Effects of variable viscosity and thermal conductivity on unsteady free convection heat and mass transfer MHD flow of micropolar fluid with constant heat flux through porous medium, Int. J. Computer Appl. $8,0975-8887$.

Uddin, M.J., Bég, O. Anwar, Ghose, P.K. and Ismael, A.I.M., 2016. Numerical study of non-Newtonian nanofluid transport in a porous medium with multiple convective boundary conditions and nonlinear thermal radiation effects, Int. J. Num. Meth. Heat Fluid Flow, 26 (5) 1-25.

Uddin, M.J., Khan, W.A., Ismail, A.I.M. and Bég, O. Anwar, 2016. Computational study of three-dimensional stagnation point nanofluid bioconvection flow on a moving surface with anisotropic slip and thermal jump effect, ASME J. Heat Trans. 138(10):104502104502-7.

Uesawa, S., Koizumi, Y., Shibata, M. and Yoshida, H., 2017. Saturated pool nucleate boiling on heat transfer surface with deposited sea salts, ASME J. Nucl. Rad. Sci. 3, 041002 .

Vocale, P., Morini, G. L. and Spiga, M., 2015. Convective heat transfer in elliptical microchannels under slip flow regime and H1 boundary conditions, ASME J. Heat Trans. 138(4):044502-044502-7.

Zueco, J., Bég, O. Anwar and Lopez-Ochoa, L.M., 2011. Effects of thermophoresis particle deposition and of the thermal conductivity in a porous plate with dissipative heat and mass transfer, Acta Mech. Sinica, 27, 389-398. 